\title{
A Similarity Degree Characterization of Nuclear $C^{*}$-algebras
}

Gilles PISIER*

\begin{abstract}
We show that a $C^{*}$-algebra $A$ is nuclear iff there is a number $\alpha<3$ and a constant $K$ such that, for any bounded homomorphism $u: A \rightarrow B(H)$, there is an isomorphism $\xi: H \rightarrow H$ satisfying $\left\|\xi^{-1}\right\|\|\xi\| \leq K\|u\|^{\alpha}$ and such that $\xi^{-1} u(.) \xi$ is a *-homomorphism. In other words, an infinite dimensional $A$ is nuclear iff its length (in the sense of our previous work on the Kadison similarity problem) is equal to 2 .

In 1955, Kadison [14] formulated the following conjecture: any bounded homomorphism $u: A \rightarrow B(H)$, from a $C^{*}$-algebra into the algebra $B(H)$ of all bounded operators on a Hilbert space $H$, is similar to a $*$-homomorphism, i.e. there is an invertible operator $\xi: H \rightarrow H$ such that $x \rightarrow \xi u(x) \xi^{-1}$ satisfies $\xi u\left(x^{*}\right) \xi^{-1}=\left(\xi u(x) \xi^{-1}\right)^{*}$ for all $x$ in $A$. This conjecture remains unproved, although many partial results are known (see [4], [10]). In particular, by [10], we know that $u$ is similar to a $*$-homomorphism iff it is completely bounded (c.b. in short) in the sense of e.g. [17] or [20] (to which we refer for background on c.b. maps). Moreover, we have

$$
\|u\|_{c b}=\inf \left\{\|\xi\|\left\|\xi^{-1}\right\|\right\}
$$

where the infimum runs over all invertible $\xi$ such that $\xi u(\cdot) \xi^{-1}$ is a $*$-homomorphism. Recall that, by definition, $\|u\|_{c b}=\sup _{n \geq 1}\left\|u_{n}\right\|$ where $u_{n}: M_{n}(A) \rightarrow$ $M_{n}(B(H))$ is the mapping taking $\left[a_{i j}\right]$ to $\left[u\left(a_{i j}\right)\right]$. Thus Kadison's conjecture

Communicated by H. Okamoto. Received July 17, 2005.

2000 Mathematics Subject Classification(s): Primary 46 L06; Seconday 46L07, 46L57.

* Partially supported by NSF grant No. 0200690.

Department of Mathematics, Texas A\&M University, College Station, TX 77843-3368, U. S. A. and Université Paris VI, Equipe d'Analyse, Case 186, 75252, Paris Cedex 05, France.

e-mail: pisier@math.tamu.edu
\end{abstract}

(C) 2006 Research Institute for Mathematical Sciences, Kyoto University. All rights reserved. 
is equivalent to the validity of the implication $\|u\|<\infty \Rightarrow\|u\|_{c b}<\infty$. In [18], the author proved that if a $C^{*}$-algebra $A$ verifies Kadison's conjecture, then there is a number $\alpha$ for which there exists a constant $K$ so that any bounded homomorphism $u: A \rightarrow B(H)$ satisfies $\|u\|_{c b} \leq K\|u\|^{\alpha}$. Moreover, the smallest number $\alpha$ with this property is an integer denoted by $d(A)$ (and $\alpha=d(A)$ itself satisfies the property).

An analogous parameter can be defined for a discrete group $G$ and it is proved in [18] that $G$ is amenable iff $d(G) \leq 2$. The main result of this note is the analogous equivalence for $C^{*}$-algebras: a $C^{*}$-algebra $A$ is nuclear (or equivalently amenable, see below) iff $d(A) \leq 2$. In [18], we could only prove a partial result in this direction.

Let $A, B$ be $C^{*}$-algebras. Let \|\|$_{\alpha}$ be a $C^{*}$-norm on their algebraic tensor product, denoted by $A \otimes B$; as usual, $A \otimes_{\alpha} B$ then denotes the $C^{*}$-algebra obtained by completing $A \otimes B$ with respect to \|\|$_{\alpha}$. By classical results (see [24]) the set of $C^{*}$-norms admits a minimal and a maximal element denoted respectively by $\|\cdot\|_{\min }$ and $\|\cdot\|_{\max }$. Then $A$ is called nuclear if for any $B$ we have $A \otimes_{\min }$ $B=A \otimes_{\max } B$, or equivalently $\|x\|_{\min }=\|x\|_{\max }$ for any $x$ in $A \otimes B$. We refer the reader to [24], [15] for more information on nuclear $C^{*}$-algebras. We note in particular that by results due to Connes and Haagerup ([7], [8]), a $C^{*}$-algebra is nuclear iff it is amenable as a Banach algebra (in B.E. Johnson's sense).

The main result of this note is as follows.

Theorem 1. The following properties of $C^{*}$-algebra $A$ are equivalent:

(i) A is nuclear.

(ii) There are $\alpha<3$ and a constant $K$ such that any bounded homomorphism $u: A \rightarrow B(H)$ satisfies $\|u\|_{c b} \leq K\|u\|^{\alpha}$.

(iii) Same as (ii) with $K=1$ and $\alpha=2$.

The implication (i) $\Rightarrow$ (iii) is well known (see [2], [4]).

In the terminology of [18], the similarity degree $d(A)$ is the smallest $\alpha$ for which the property considered in (ii) above is satisfied. It is proved in [18] that $d(A)$ is always an integer identified as the smallest length of a specific kind of factorization for matrices with entries in $A$.

With this terminology, the preceding theorem means that $A$ is nuclear iff $d(A) \leq 2$. In the infinite dimensional case, $d(A)>1$ hence $A$ is nuclear iff $d(A)=2$.

In his work on derivations (see [4] and [5]) Erik Christensen isolated the following property $D_{k}$ for a $C^{*}$-algebra. Here $k$ is any number $\geq 1 / 2$. A $C^{*}$ algebra $A$ has property $D_{k}$ if for any $H$, any representation $\pi: A \rightarrow B(H)$, and 
any $T$ in $B(H)$ the derivation $\delta_{T}: A \rightarrow B(H)$ defined by $\delta_{T}(a)=\pi(a) T-T \pi(a)$ satisfies

$$
\left\|\delta_{T}\right\|_{c b} \leq 2 k\left\|\delta_{T}\right\|
$$

With this terminology, Theorem 1 implies the following:

Corollary 2. Let $A$ be $a C^{*}$-algebra. The following assertions are equivalent.

(i) A is nuclear.

(ii) A satisfies property $D_{k}$ for some $k<3 / 2$.

(iii) A satisfies property $D_{1}$.

Proof. Here again the fact that (i) $\Rightarrow$ (iii) is well known (see [2], [4]). The equivalence between the similarity problem and the derivation problem was established by Kirchberg in [16]. Refining Kirchberg's estimates, the author proved in [18] (see also [20, p. 139]) that property $D_{k}$ implies that the similarity degree $d(A)$ is at most $2 k$. Thus (ii) $\Rightarrow$ (i) follows from the corresponding implication in Theorem 1.

The main point in Theorem 1 is (ii) $\Rightarrow$ (i). In our previous work, we could only prove that (ii) implies that $A$ is "semi-nuclear," i.e. that whenever a representation $\pi: A \rightarrow B(H)$ generates a semifinite von Neumann algebra, the latter is injective. In this note, we show that the semifiniteness assumption is not needed. We use the same starting point as in [18], but we feel the idea of the present proof is more transparent than the one in [18]. In particular, we will use the following result which is part of Th.2.9 in [19] (obtained independently in [6]), but the latter is inspired by and closely related to Haagerup's Th. 2.1 in $[9]$.

Theorem 3. Let $N \subset B(H)$ be a von Neumann algebra. Then $N$ is injective iff there is a constant $C$ such that, for all $n$, if elements $x_{i}$ in $N(i=$ $1, \ldots, n)$ admit a decomposition $x_{i}=\alpha_{i}+\beta_{i}$ with $\alpha_{i}, \beta_{i} \in B(H)$ such that $\left\|\sum \alpha_{i}^{*} \alpha_{i}\right\| \leq 1$ and $\left\|\sum \beta_{i} \beta_{i}^{*}\right\| \leq 1$, then there is a decomposition $x_{i}=a_{i}+b_{i}$ with $a_{i}, b_{i} \in N$ such that $\left\|\sum a_{i}^{*} a_{i}\right\| \leq C^{2}$ and $\left\|\sum b_{i} b_{i}^{*}\right\| \leq C^{2}$.

The preceding statement can be viewed as the analogue for von Neumann algebras of the characterization of amenable discrete groups obtained in [27] (see also [1]).

Our main (somewhat) new ingredient is as follows. 
Theorem 4. Let $M \subset B(H)$ be a von Neumann algebra with a cyclic vector. Let $y_{1}, \ldots, y_{n}$ in $M^{\prime}$ be such that for any $x_{1}, \ldots, x_{n}$ in $M$ we have

$$
\left\|\sum x_{i} y_{i}\right\| \leq \max \left\{\left\|\sum x_{i}^{*} x_{i}\right\|^{1 / 2},\left\|\sum x_{i} x_{i}^{*}\right\|^{1 / 2}\right\} .
$$

Then there is a decomposition

$$
y_{i}=a_{i}+b_{i}
$$

with $a_{i}, b_{i}$ in $M^{\prime}$ such that

$$
\left\|\sum a_{i} a_{i}{ }^{*}\right\| \leq 1 \quad \text { and } \quad\left\|\sum b_{i}{ }^{*} b_{i}\right\| \leq 1 .
$$

Proof. We follow a well known kind of argument with roots in [9]; see also [23] and the proof of a theorem due to Kirchberg as presented in [20, §14] that we will follow closely below.

Recall that the "row and column" operator spaces $R_{n} \subset M_{n}$ and $C_{n} \subset M_{n}$ are defined by:

$$
R_{n}=\operatorname{span}\left[e_{1 i} \mid 1 \leq i \leq n\right] \quad C_{n}=\operatorname{span}\left[e_{i 1} \mid 1 \leq i \leq n\right]
$$

Let $\Delta_{n} \subset C_{n} \oplus R_{n}$ be the operator space spanned by $\delta_{i}=e_{i 1} \oplus e_{1 i}(i=$ $1,2, \ldots, n)$. Our assumption means that the linear map

$$
v: \Delta_{n} \otimes_{\min } M \rightarrow B(H)
$$

defined by

$$
v\left(\sum \delta_{i} \otimes x_{i}\right)=\sum x_{i} y_{i}
$$

satisfies $\|v\| \leq 1$. (Indeed, it is easy to check that the majorant in (1) is equal to $\left.\left\|\sum \delta_{i} \otimes x_{i}\right\|_{\min }.\right)$

Since $v$ is clearly a two-sided $M$-module map and $M$ has a cyclic vector, it follows by [22] (and unpublished work of Haagerup) that $\|v\|_{c b}=\|v\| \leq 1$.

Therefore, by a result due to Wittstock [26] (see also [23]), $v$ can be extended to a two-sided $M$-module map $\tilde{v}:\left[C_{n} \oplus R_{n}\right] \otimes_{\min } M \rightarrow B(H)$ with $\|\tilde{v}\|_{c b} \leq 1$. Let $a_{i}=\tilde{v}\left(\left[e_{i 1} \oplus 0\right] \otimes 1\right)$ and $b_{i}=\tilde{v}\left(\left[0 \oplus e_{1 i}\right] \otimes 1\right)$. Then we have clearly $\left\|\sum a_{i} a_{i}{ }^{*}\right\|^{1 / 2} \leq\|\tilde{v}\|_{c b} \leq 1$ and similarly $\left\|\sum b_{i}{ }^{*} b_{i}\right\|^{1 / 2} \leq 1$. Moreover, since $\tilde{v}$ is an $M$-module map, for any $m$ in $M$ and any z in $C_{n} \oplus R_{n}$, we must have $m . \tilde{v}(z \otimes 1)=\tilde{v}(z \otimes m)=\tilde{v}(z \otimes 1) . m$ and hence $\tilde{v}(z \otimes 1) \in M^{\prime}$. Thus $a_{i}$ and $b_{i}$ are in $M^{\prime}$. 
Remark 1. It is easy to see that the preceding result fails without the cyclicity assumption: Just consider the case $M=\mathbb{C}$ and $M^{\prime}=B(H)$ with $\operatorname{dim}(H)=\infty$.

Remark 2. The same proof gives a criterion for a map $u: E \rightarrow M^{\prime}$ defined on a subspace $E \subset A$ of a general $C^{*}$-algebra $A$ to admit an extension $\tilde{u}: A \rightarrow M^{\prime}$ with $\|\tilde{u}\|_{d e c} \leq 1$. This is essentially the same as Kirchberg's [20, Th. 14.6].

Remark 3. The above Theorem 4 may be viewed as an analogue for the operator space $R_{n}+C_{n}$ of Haagerup's [9, Lemma 3.5] devoted to the operator space $\ell_{1}^{n}$ equipped with its maximal structure, in the Blecher-Paulsen sense (see e.g. $[20, \S 3])$. Note that while he decomposes into products, we decompose into sums.

Remark 4. Let $\left(E_{0}, E_{1}\right)$ be a compatible pair of operator spaces in the sense of $[20, \S 2.7]$. Then Remark 2 gives a sufficient criterion for a map $u$ : $E_{0}+$ $E_{1} \rightarrow M^{\prime}$ to admit a decomposition $u=u_{0}+u_{1}$ with $u_{0}: E_{0} \rightarrow M^{\prime}$ and $u_{1}: E_{1} \rightarrow M^{\prime}$ satisfying $\left\|u_{0}\right\|_{c b} \leq 1$ and $\left\|u_{1}\right\|_{c b} \leq 1$. Assume that $E_{0} \subset A_{0}$ and $E_{1} \subset A_{1}$, where $A_{0}, A_{1}$ are $C^{*}$-algebras, then this criterion actually ensures that there are extensions

$$
\tilde{u}_{0}: A_{0} \rightarrow M^{\prime} \quad \text { and } \quad \tilde{u}_{1}: A_{1} \rightarrow M^{\prime}
$$

with $\left\|\tilde{u}_{0}\right\|_{\text {dec }} \leq 1$ and $\left\|\tilde{u}_{1}\right\|_{\text {dec }} \leq 1$. In that formulation, the converse also holds up to a numerical factor 2 . Note that, in the special case of interest to us, when $E_{0}=C$ and $E_{1}=R$, then we can take $A_{0}, A_{1}$ equal to $K\left(\ell_{2}\right)$ (hence nuclear) so that the min and max norms are identical on $\left(A_{0} \oplus A_{1}\right) \otimes M$.

Notation. Let $A \subset B(H)$ be any $C^{*}$-subalgebra. For any $x_{1}, \ldots, x_{n}$ and $y_{1}, \ldots, y_{n}$ in $A$, we denote

$$
\begin{aligned}
& \left\|\left(x_{j}\right)\right\|_{R \cap C}=\max \left\{\left\|\sum x_{j}^{*} x_{j}\right\|^{1 / 2},\left\|\sum x_{j} x_{j}^{*}\right\|^{1 / 2}\right\} \\
& \left\|\left(y_{j}\right)\right\|_{R+C}=\inf \left\{\left\|\sum \alpha_{j}^{*} \alpha_{j}\right\|^{1 / 2}+\left\|\sum \beta_{j} \beta_{j}^{*}\right\|^{1 / 2}\right\},
\end{aligned}
$$

where the infimum runs over all $\alpha_{j}, \beta_{j}$ in $B(H)$ such that $y_{j}=\alpha_{j}+\beta_{j}$. Note that, by the injectivity of $B(H)$, the definition of $\left\|\left(y_{j}\right)\right\|_{R+C}$ does not really depend on the choice of $H$ or of the embedding $A \subset B(H)$. The corresponding fact for $\left\|\left(x_{j}\right)\right\|_{R \cap C}$ is obvious. 
Corollary 5. Let $M \subset B(H)$ be a von Neumann algebra. Then $M$ is injective iff there is a constant $C$ such that, for all $n$, all $x_{1}, \ldots, x_{n}$ in $M$ and $y_{1}, \ldots, y_{n}$ in $M^{\prime}$, we have

$$
\left\|\sum x_{i} y_{i}\right\| \leq C\left\|\left(x_{i}\right)\right\|_{R \cap C}\left\|\left(y_{i}\right)\right\|_{R+C}
$$

Proof. If $M$ has a cyclic vector, then this follows immediately from Theorems 3 and 4 and the well known fact that $M^{\prime}$ is injective iff $M$ is injective (see [25, p. 174]). Now assume that $M$ has a finite cyclic set, i.e. there are $\xi_{1}, \ldots, \xi_{N}$ in $H$ such that $M \xi_{1}+\cdots+M \xi_{N}$ is dense in $H$. Then the vector $\left(\xi_{1}, \ldots, \xi_{N}\right)$ in $H^{N}$ is cyclic for $M_{N}(M) \subset M_{N}(B(H))$. Moreover, it is easy to check that (4) remains true for $M_{N}(M)$ but with $C$ replaced by a constant $C(N)$ (possibly unbounded when $N$ grows). Nevertheless, by the first part of the proof it follows that $M_{N}(M)$ is injective and hence, a fortiori, $M$ is injective.

In the general case, let $\left\{\xi_{i} \mid i \in I\right\}$ be a dense subset of $H$. For any finite subset $J \subset I$, let $H_{J}$ be the closure of

$$
\left\{\sum_{j \in J} a_{j}\left(\xi_{j}\right) \mid a_{j} \in M\right\} .
$$

Note that $H_{J}$ is an invariant subspace for $M$, so that (since $M$ is self-adjoint) the orthogonal projection $P_{J}: H \rightarrow H_{J}$ belongs to $M^{\prime}$. Let $\pi_{J}(a)$ denote the restriction of $a$ to $H_{J}$. Then $\pi_{J}: M \rightarrow B\left(H_{J}\right)$ is a normal representation, $\pi_{J}(M)$ admits a finite cyclic set (namely $\left\{\xi_{i} \mid i \in J\right\}$ ), and it is easy to check that our assumption (4) is still verified by $\pi_{J}(M)$ on $H_{J}$.

Thus, by the first part of the proof, $\pi_{J}(M)$ is injective. This clearly implies that the von Neumann algebra $M_{J} \subset B(H)$ generated by $P_{J} M$ and $I-P_{J}$ also is injective. Finally, since $M$ is the weak-* closure of the directed union of the $M_{J}$ 's, we conclude that $M$ itself is injective.

Conversely, if $M$ injective then, by Remark 5 below, (4) holds with $C=1$.

Remark 5. Let $M \subset B(H)$ be an injective von Neumann algebra, so that there is a projection $P: B(H) \rightarrow M^{\prime}$ with $\|P\|_{c b}=1$. Then $M$ satisfies (4) with $C=1$. To see this, assume $y_{i} \in M^{\prime}$ and $\left\|\left(y_{i}\right)\right\|_{R+C}<1$, so that $y_{i}=\alpha_{i}+\beta_{i}$ with $\left\|\Sigma \alpha_{i}^{*} \alpha_{i}\right\|^{1 / 2}+\left\|\Sigma \beta_{i} \beta_{i}^{*}\right\|^{1 / 2}<1$. Then $y_{i}=a_{i}+b_{i}$ with $a_{i}, b_{i} \in M^{\prime}$ satisfying

$$
\left\|\sum a_{i}^{*} a_{i}\right\|^{1 / 2}+\left\|\sum b_{i} b_{i}^{*}\right\|^{1 / 2} \leq\|P\|_{c b}=1 .
$$

Indeed, $a_{i}=P \alpha_{i}$ and $b_{i}=P \beta_{i}$ clearly verify this. 
Then for any $x_{1}, \ldots, x_{n}$ in $M$ we have by Cauchy-Schwarz

$$
\left\|\sum x_{i} a_{i}\right\| \leq\left\|\sum x_{i} x_{i}^{*}\right\|^{1 / 2}\left\|\sum a_{i}^{*} a_{i}\right\|^{1 / 2}
$$

and

$$
\left\|\sum b_{i} x_{i}\right\| \leq\left\|\sum x_{i}^{*} x_{i}\right\|^{1 / 2}\left\|\sum b_{i} b_{i}^{*}\right\|^{1 / 2}
$$

therefore, since

$$
\left\|\sum x_{i} y_{i}\right\| \leq\left\|\sum x_{i} a_{i}\right\|+\left\|\sum b_{i} x_{i}\right\|
$$

we obtain finally

$$
\left\|\sum x_{i} y_{i}\right\| \leq\left\|\left(x_{i}\right)\right\|_{R \cap C}\left\|\left(y_{i}\right)\right\|_{R+C}
$$

We will also use:

Theorem 6 ([18]). A unital operator algebra A satisfies property (ii) in Theorem 1 iff we have: (iv) There is a constant $K^{\prime}$ satisfying the following: for any linear map $u: A \rightarrow B(H)$ for which there are a Hilbert space $K$, bounded linear maps $v_{1}, w_{1}$ from $A$ to $B(K, H)$ and $v_{2}, w_{2}$ from $A$ to $B(H, K)$ such that

$$
\forall a, b \in A \quad u(a b)=v_{1}(a) v_{2}(b)+w_{1}(a) w_{2}(b)
$$

we have

$$
\|u\|_{c b} \leq K^{\prime}\left(\left\|v_{1}\right\|\left\|v_{2}\right\|+\left\|w_{1}\right\|\left\|w_{2}\right\|\right)
$$

Remark. Note that (5) implies that the bilinear map $(a, b) \rightarrow u(a b)$ is c.b. on $\max (A) \otimes_{h} \max (A)$ with c.b. norm $\leq K^{\prime}\left(\left\|v_{1}\right\|\left\|v_{2}\right\|+\left\|w_{1}\right\|\left\|w_{2}\right\|\right)$. Thus, Theorem 6 follows from the case $d=2$ of [18, Th. 4.2].

Another ingredient is the following Lemma which can be derived from [13] or from the more recent paper [21].

Lemma 7. Let $E$ be a finite dimensional operator space and let $A$ be a $C^{*}$-algebra. Assume that $E$ is a "maximal" operator space (equivalently that $E^{*}$ is a minimal one). Then for any c.b. map $u: A \rightarrow E$ we have

$$
\forall n \forall a_{1}, \ldots, a_{n} \in A \quad \forall \xi_{i} \in E^{*}
$$




$$
\begin{aligned}
\left|\sum\left\langle u\left(a_{j}\right), \xi_{j}\right\rangle\right| \leq & C\|u\|_{c b}\left(\left\|\sum a_{j}^{*} a_{j}\right\|^{1 / 2}+\left\|\sum a_{j} a_{j}^{*}\right\|^{1 / 2}\right) \\
& \cdot \sup _{x \in E}\left(\sum\left|\xi_{j}(x)\right|^{2}\right)^{1 / 2}
\end{aligned}
$$

where $C$ is a numerical constant.

Proof. We may apply [13, Th. 1.4], arguing as in [18, Lemma 6.3] (using [19, Th. 17.13] to remove the exactness assumption) this yields (6) with $C=2$. Or we may invoke [21, Th. 0.3] taking into account [21, Lemma 2.3] (to remove the exactness assumption) and then we again obtain (6) with $C=2$.

For the convenience of the reader, we reproduce here the elementary Lemma 8 from [18].

Lemma 8. Let $\left(e_{i}\right)$ be the canonical basis of the operator space $\max \left(\ell_{2}\right)$. Let $H$ be any Hilbert space and let $X$ be either $B(\mathbb{C}, H)$ or $B\left(H^{*}, \mathbb{C}\right)$, or equivalently let $X$ be either the column Hilbert space or the row Hilbert space. Then for all $x_{1}, \ldots, x_{n}$ in $X$ we have

$$
\left\|\sum_{1}^{n} x_{i} \otimes e_{i}\right\|_{X \otimes_{\min } \max \left(\ell_{2}\right)} \leq\left(\sum\left\|x_{i}\right\|^{2}\right)^{1 / 2} .
$$

Proof. Assume $X=B(\mathbb{C}, H)$ or $B\left(H^{*}, \mathbb{C}\right)$. We identify $X$ with $H$ as a vector space. Let $\left(\delta_{m}\right)$ be an orthonormal basis in $H$. Observe that for any finite sequence $a_{m}$ in $B\left(\ell_{2}\right)$ we have in both cases

$$
\left\|\sum \delta_{m} \otimes a_{m}\right\|_{\min } \leq\left(\sum\left\|a_{m}\right\|^{2}\right)^{1 / 2}
$$

whence we have, for any $x_{1}, \ldots, x_{n}$ in $X$,

$$
\begin{aligned}
\left\|\sum x_{i} \otimes e_{i}\right\| & =\left\|\sum_{m} \delta_{m} \otimes \sum_{i}\left\langle x_{i}, \delta_{m}\right\rangle e_{i}\right\| \\
& \leq\left(\sum_{m}\left\|\sum_{i}\left\langle x_{i}, \delta_{m}\right\rangle e_{i}\right\|^{2}\right)^{1 / 2} \\
& =\left(\sum_{m, i}\left|\left\langle x_{i}, \delta_{m}\right\rangle\right|^{2}\right)^{1 / 2}=\left(\sum_{i}\left\|x_{i}\right\|^{2}\right)^{1 / 2} .
\end{aligned}
$$


Proof of Theorem 1. As we already observed, it suffices to show that (ii) implies that $A$ is nuclear. Let $\pi: A \rightarrow B(H)$ be a representation and let $M=\pi(A)^{\prime \prime}$. Using Theorem 6 and Corollary 5, we will show that (ii) implies that $M$ is injective. By the well known results of Choi-Effros and Connes (see [3]), this implies that $A$ is nuclear. Since $\pi(A) \simeq A / \operatorname{ker}(\pi)$ is a quotient of $A$, it obviously inherits the property (ii). Thus we may as well replace $\pi(A)$ by $A$ : we assume $A \subset B(H)$ and let $M=A^{\prime \prime}$. It suffices to show that $M$ is injective.

Claim. We claim that for any $x_{1}, \ldots, x_{n}$ in $M$ and $y_{1}, \ldots, y_{n}$ in $M^{\prime}$ we have

$$
\left\|\sum x_{j} y_{j}\right\| \leq 4 K^{\prime} C\left\|\left(x_{j}\right)\right\|_{R \cap C}\left\|\left(y_{j}\right)\right\|_{R+C} .
$$

Note: It may be worthwhile for the reader to note that $\left\|\left(y_{j}\right)\right\|_{R+C}$ is (up to a factor 2) in operator space duality with $\left\|\left(x_{j}\right)\right\|_{R \cap C}$, namely if we set

$$
\left\|\left(y_{j}\right)\right\|=\sup \left\{\left\|\sum x_{j} \otimes y_{j}\right\|_{\min }\right\}
$$

where the sup runs over all $\left(x_{j}\right)$ in $B\left(\ell_{2}\right)$ such that $\left\|\left(x_{j}\right)\right\|_{R \cap C} \leq 1$, then we have (see e.g. [12])

$$
\left\|\left(y_{j}\right)\right\|\left|\leq\left\|\left(y_{j}\right)\right\|_{R+C} \leq 2\right|\left\|\left(y_{j}\right)\right\| \| .
$$

To prove (7) we introduce the operator space $E=\max \left(\ell_{2}^{n}\right)$, that is $n$ dimensional Hilbert space equipped with its "maximal" operator space structure in the Blecher-Paulsen sense (see $[20, \S 3])$. Let us now fix an $n$-tuple $\left(y_{j}\right)$ in $M^{\prime}$ such that $\left\|\left(y_{j}\right)\right\|_{R+C}<1$. In addition, we fix $\xi, \eta$ in the unit sphere of $H$. Then we define a linear map $u: M \rightarrow E$ as follows:

$$
u(x)=\sum_{j}\left\langle x y_{j} \xi, \eta\right\rangle e_{j}
$$

where $e_{j}$ is the canonical basis of $\ell_{2}^{n}$. We will assume that $E \subset B(K)$ completely isometrically. The reader may prefer to consider instead of $u$, the bilinear form $(x, \xi) \rightarrow\langle u(x), \xi\rangle$ defined on $M \times E^{*}$ where $E^{*}$ is now $\ell_{2}^{n}$ equipped with its "minimal" (or commutative) operator space structure obtained by embedding it isometrically into a commutative $C^{*}$-algebra. We will now apply Theorem 6 to $u$.

Since we assume $\left\|\left(y_{j}\right)\right\|_{R+C}<1$, we can write

$$
y_{j}=\alpha_{j}+\beta_{j}
$$

with $\left\|\Sigma \alpha_{j}^{*} \alpha_{j}\right\|<1$ and $\left\|\Sigma \beta_{j} \beta_{j}^{*}\right\|<1$. Note that, since $y_{j} \in M^{\prime}$, for all $a, b$ in $M$ we have

$$
a b y_{j}=a y_{j} b
$$


and hence

$$
u(a b)=V(a, b)+W(a, b)
$$

where

$$
\begin{aligned}
V(a, b) & =\sum\left\langle a \alpha_{j} b \xi, \eta\right\rangle e_{j} \\
W(a, b) & =\sum\left\langle a \beta_{j} b \xi, \eta\right\rangle e_{j} .
\end{aligned}
$$

We now claim that we can write for all $a, b$ in $M$

$$
V(a, b)=v_{1}(a) v_{2}(b) \quad \text { and } \quad W(a, b)=w_{1}(a) w_{2}(b)
$$

where

$$
\begin{aligned}
& v_{1}: \quad M \rightarrow B(H \otimes K, K), \quad w_{1}: \quad M \rightarrow B(H \otimes K, K) \\
& v_{2}: M \rightarrow B(K, H \otimes K), \quad w_{2}: M \rightarrow B(K, H \otimes K)
\end{aligned}
$$

are linear maps all with norm $\leq 1$.

Indeed, let us set for $h \in H, k \in K$

$$
\begin{aligned}
v_{1}(a)(h \otimes k) & =\sum_{j}\left\langle a \beta_{j} h, \eta\right\rangle e_{j} k \\
w_{1}(a)(h \otimes k) & =\langle a h, \eta\rangle k \\
v_{2}(b)(k) & =b \xi \otimes k \\
w_{2}(b)(k) & =\sum_{j} \alpha_{j} b \xi \otimes e_{j} k .
\end{aligned}
$$

Then, it is easy to check (8). Also, we have trivially

$$
\begin{aligned}
\left\|w_{1}(a)\right\| & =\left\|a^{*} \eta\right\| \leq\|a\| \\
\left\|v_{2}(b)\right\| & =\|b \xi\| \leq\|b\| .
\end{aligned}
$$

Moreover, by Lemma 8 we have

$$
\begin{aligned}
\left\|v_{1}(a)\right\|^{2} & \leq \sum_{j}\left\|\beta_{j}^{*} a^{*} \eta\right\|^{2}=\left\langle\sum \beta_{j} \beta_{j}^{*} a^{*} \eta, a^{*} \eta\right\rangle \\
& \leq\left\|a^{*} \eta\right\|^{2} \leq\|a\|^{2} \\
\left\|w_{2}(b)\right\|^{2} & \leq \sum_{j}\left\|\alpha_{j} b \xi\right\|^{2}=\left\langle\sum \alpha_{j}^{*} \alpha_{j} b \xi, b \xi\right\rangle \\
& \leq\|b \xi\|^{2} \leq\|b\|^{2} .
\end{aligned}
$$

By Theorem 6, it follows that

$$
\left\|u_{\mid A}\right\|_{c b} \leq 2 K^{\prime}
$$


Since $u: M \rightarrow B(K)$ is clearly normal (i.e. $\sigma\left(M, M_{*}\right)$ continuous) and since $A$ is $\sigma\left(M, M_{*}\right)$ dense in $M$, we clearly have (by the Kaplansky density theorem)

$$
\|u\|_{c b}=\left\|u_{\mid A}\right\|_{c b} \leq 2 K^{\prime} .
$$

Then by Lemma 7 , applied with $\xi_{j}$ biorthogonal to $e_{j}$, we have

$$
\forall n \forall x_{1}, \ldots, x_{n} \in M \quad\left|\left\langle\sum x_{j} y_{j} \xi, \eta\right\rangle\right| \leq 4 K^{\prime} C\left\|\left(x_{j}\right)\right\|_{R \cap C} .
$$

Hence, taking the supremum over $\xi, \eta$ and using homogeneity, we obtain the claimed inequality (7). Then, by Corollary $5, M$ is injective.

Remark. Since Lemma 4 actually holds whenever $A$ is an exact operator space (with $C$ replaced by twice the exactness constant [13], [21]), the proof of Theorem 1 shows that any unital, exact (non selfadjoint) operator algebra $A \subset B(H)$ with $d(A) \leq 2$ in the sense of [18] satisfies (4) for some $C$.

The preceding arguments establish the following result of independent interest.

Theorem 9. $\quad A C^{*}$-algebra $A$ is nuclear iff for any $C^{*}$-algebra $B$ there is a constant $C$ such that, for all $n$, all $x_{1}, \ldots, x_{n}$ in $A$ and all $y_{1}, \ldots, y_{n}$ in $B$ we have

$$
\left\|\sum x_{i} \otimes y_{i}\right\|_{\max } \leq C\left\|\left(x_{i}\right)\right\|_{R \cap C}\left\|\left(y_{i}\right)\right\|_{R+C}
$$

Proof. Let $\pi: A \rightarrow B(H)$ be a representation. Taking $B=\pi(A)^{\prime}$ (and using the fact that the set of $n$-tuples $\left(x_{i}\right)$ in $A^{* *}$ with $\left\|\left(x_{i}\right)\right\|_{R \cap C} \leq 1$ is the weak-* closure of its intersection with $A^{n}$, see e.g. [20, p. 303]) we see that (9) implies (4) for $M=\pi(A)^{\prime \prime}$. Since this holds for any $\pi$, we may argue as in the preceding proof (replacing $\pi$ by $\pi_{J}$ ) to conclude that $\pi(A)^{\prime \prime}$ is injective, and hence that $A$ is nuclear. Conversely, if $A$ is nuclear it is easy to show (see Remark 5) that (9) holds with $C=1$.

Theorem 10. $A C^{*}$-algebra $A$ is nuclear iff for any $C^{*}$-algebra $B$ there is a constant $C$ such that for all $n$, all $x_{1}, \ldots, x_{n}$ in $A$ and all $y_{1}, \ldots, y_{n}$ in $B$ we have

$$
\left\|\sum x_{i} \otimes y_{i}\right\|_{\max } \leq C\left\|\sum x_{i} \otimes \bar{x}_{i}\right\|_{\min }^{1 / 2}\left\|\sum y_{i} \otimes \bar{y}_{i}\right\|_{\min }^{1 / 2}
$$

where the min norms are relative to $A \otimes \bar{A}$ and $B \otimes \bar{B}$. 
Proof. It is known (see $[19,(2.12)]$ ) that $\left\|\Sigma x_{i} \otimes \bar{x}_{i}\right\|_{\text {min }}^{1 / 2} \leq\left\|\left(x_{i}\right)\right\|_{R \cap C}$. Thus, arguing as above, we find that for any representation $\pi: A \rightarrow B(H)$ the von Neumann algebra $M=\pi(A)^{\prime \prime}$ satisfies the following: if $y_{1}, \ldots, y_{n}$ in $M^{\prime}$ are such that $\left\|\Sigma y_{i} \otimes \bar{y}_{i}\right\|_{\text {min }}<1$, then there are $a_{i}, b_{i}$ in $M^{\prime}$ with $y_{i}=a_{i}+b_{i}$ such that $\left\|\Sigma a_{i}^{*} a_{i}\right\|^{1 / 2}<C$ and $\left\|\Sigma b_{i} b_{i}^{*}\right\|^{1 / 2}<C$. By [19, Th. 2.9], this ensures that $M^{\prime}$ is injective, and hence $A$ is nuclear.

Remark $6 . \quad$ Note however that by [11] the inequality

$$
\left\|\sum x_{i} \otimes \bar{x}_{i}\right\|_{\max }^{1 / 2} \leq C\left\|\sum x_{i} \otimes \bar{x}_{i}\right\|_{\min }^{1 / 2}
$$

characterizes the weak expectation property, which is strictly more general than nuclearity.

Remark. It would be nice to know exactly which families of pairs of operator spaces in duality $\left(F_{n}, F_{n}^{*}\right)$ can be used instead of $F_{n}=R_{n} \cap C_{n}$ or $F_{n}=O H_{n}$ to characterize nuclearity (or injectivity) analogously to the above Theorems 9 and 10 (note that $F_{n}=R_{n}$ or $F_{n}=C_{n}$ obviously do not work).

We will say that a function $f: \mathbb{N} \rightarrow \mathbb{R}_{+}$is "slowly growing" if, for any $\varepsilon>0$, there is a constant $C_{\varepsilon}$ such that $f(n) \leq C_{\varepsilon} n^{\varepsilon}$ for all $n \geq 1$.

The rest of the paper is devoted to a technical refinement, based on the following observation: assume that in Theorem 3 the constant $C$ depends on $n$, i.e. $C=C(n)$ but that it is "slowly growing". Then $N$ is injective.

Indeed, as for Theorem 3 , this observation follows from the same argument as for [19, Th. 2.9], itself based on [9]. Recall Haagerup's characterization of finite injective von Neumann algebras $([9$, Lemma 2.2]): $N$ is finite and injective iff for any $n$-tuple $\left(u_{i}\right)$ of unitaries and any central projection $p$ in $N$ we have

$$
n=\left\|\sum p u_{i} \otimes \overline{p u_{i}}\right\|
$$

Actually, for this to hold it suffices that there exists a slowly growing function $C(n)$ such that for any $n$-tuple $\left(u_{i}\right)$ of unitaries and any central projection $p$ in $N$ we have

$$
n \leq C(n)\left\|\sum p u_{i} \otimes \overline{p u_{i}}\right\| .
$$

Indeed, if we set $t=\sum p u_{i} \otimes \overline{p u_{i}}$ and if we apply the preceding inequality to $\left(t^{*} t\right)^{m}$, take the $m$-th root and let $m$ go to infinity, then we find that (11) implies (10) (a similar trick appears in [9, Lemma 2.2]). Given that this is true, the above observation can be deduced, first in the case when $N$ is semifinite, 
and then in the general case, from the finite case by the same basic reasoning as in [9].

The following theorems are then easy to obtain in the same way as above.

Theorem 11. The following properties of a $C^{*}$-algebra are equivalent.

(i) A is nuclear.

(ii) There is a slowly growing function $C: \mathbb{N} \rightarrow \mathbb{R}_{+}$such that for any $n$ and any $C^{*}$-algebra $B$ we have:

$$
\forall\left(x_{i}\right) \in A^{n} \quad \forall\left(y_{i}\right) \in B^{n} \quad\left\|\sum x_{i} \otimes y_{i}\right\|_{\max } \leq C(n)\left\|\left(x_{i}\right)\right\|_{R \cap C}\left\|\left(y_{i}\right)\right\|_{R+C} .
$$

(iii) There is a slowly growing function $C: \mathbb{N} \rightarrow \mathbb{R}_{+}$such that for any $n$ and any $C^{*}$-algebra $B$, we have:

$$
\begin{aligned}
& \forall\left(x_{i}\right) \in A^{n} \quad \forall\left(y_{i}\right) \in B^{n} \\
& \left\|\sum x_{i} \otimes y_{i}\right\|_{\max } \leq C(n)\left\|\sum x_{i} \otimes \bar{x}_{i}\right\|_{\min }^{1 / 2}\left\|\sum y_{i} \otimes \bar{y}_{i}\right\|_{\min }^{1 / 2} .
\end{aligned}
$$

Corollary 12. A von Neumann algebra $M$ is injective iff there is a slowly growing function $C: \mathbb{N} \rightarrow \mathbb{R}_{+}$such that, for any $n$, any mapping $u: \Delta_{n} \rightarrow M$ admits an extension $\tilde{u}: M_{n} \oplus M_{n} \rightarrow M$ such that

$$
\|\tilde{u}\|_{c b} \leq C(n)\|u\|_{c b}
$$

Remark. Consider a map $u: E \rightarrow F$ between operator spaces. Let $\gamma(u)=\inf \left\{\|v\|_{c b}\|w\|_{c b}\right\}$ where the infimum runs over all Hilbert spaces $H$ and all possible factorizations $u=v w$ of $u$ through $B(H)$ (here $v: B(H) \rightarrow F$ and $w: E \rightarrow B(H))$. Let $M$ be a von Neumann algebra. Assume that there is a constant $C$ so that, for any $n$, any $u: R_{n} \cap C_{n} \rightarrow M$ satisfies $\gamma(u) \leq C\|u\|_{c b}$. Then, by the preceding Corollary, $M$ is injective. Actually, even if $C=C(n)$ depends on $n$, but grows slowly when $n \rightarrow \infty$, we conclude that $M$ is injective, and hence, a posteriori, we can factor through $B(H)$ any $u$ that takes values in $M$, regardless of its domain. It seems interesting to investigate which (sequences of) operator spaces have the property that they "force" injectivity like $\left\{R_{n} \cap C_{n}\right\}$. One can show that $\left\{O H_{n}\right\}$ has that property too, but obviously not $\left\{R_{n}\right\}$ or $\left\{C_{n}\right\}$, since these are themselves injective! 


\section{References}

[1] Bożejko, M., Positive definite bounded matrices and a characterization of amenable groups, Proc. Amer. Math. Soc., 95 (1985), 357-360.

[2] Bunce, J. W., The similarity problem for representations of $C^{*}$-algebras, Proc. Amer. Math. Soc., 81 (1981), 409-414.

[3] Choi, M. D. and Effros, E., Nuclear $C^{*}$-algebras and injectivity: The general case, Indiana Univ. Math. J., 26 (1977), 443-446.

[4] Christensen, E., On non self adjoint representations of operator algebras, Amer. J. Math., 103 (1981), 817-834.

[5] - Near inclusions of $C^{*}$-algebras, Acta Math., 144 (1980), 249-265.

[6] Christensen, E. and Sinclair, A., On von Neumann algebras which are complemented subspaces of $B(H)$, J. Funct. Anal., 122 (1994), 91-102.

[7] Connes, A., On the cohomology of operator algebras, J. Funct. Anal., 28 (1978), 248253.

[8] Haagerup, U., All nuclear $C^{*}$-algebras are amenable, Invent. Math., 74 (1983), 305-319.

[9] - Injectivity and decomposition of completely bounded maps, Operator algebras and their connections with topology and ergodic theory (Buşteni, 1983), 170-222, Lecture Notes in Math., 1132, Springer, Berlin, 1985.

[10] Solution of the similarity problem for cyclic representations of $C^{*}$-algebras, Ann. Math., 118 (1983), 215-240.

[11] Self-polar forms, conditional expectations and the weak expectation property for $C^{*}$-algebras, Unpublished manuscript (1995).

[12] Haagerup, U. and Pisier, G., Bounded linear operators between $C^{*}$-algebras, Duke Math. J., 71 (1993), 889-925.

[13] Junge, M. and Pisier, G., Bilinear forms on exact operator spaces and $B(H) \otimes B(H)$, Geom. Funct. Anal., 5 (1995), 329-363.

[14] Kadison, R., On the orthogonalization of operator representations, Amer. J. Math., 77 (1955), 600-620.

[15] Kadison, R. and Ringrose, J., Fundamentals of the theory of operator algebras, Vol. II, Advanced Theory, Academic Press, New-York, 1986.

[16] Kirchberg, E., The derivation and the similarity problem are equivalent, J. Operator Theory, 36 (1996), 59-62.

[17] Paulsen, V., Completely bounded maps and operator algebras, Cambridge University Press, Cambridge, 2002.

[18] Pisier, G., The similarity degree of an operator algebra, St. Petersburg Math. J., 10 (1999), 103-146.

[19] $\quad$ The operator Hilbert space $O H$, complex interpolation and tensor norms, $\mathrm{Mem}$. Amer. Math. Soc., 122 (1996), 1-103.

[20] , Introduction to Operator Space Theory, Cambridge Univ. Press, 2003.

[21] Shlyakhtenko, D. and Pisier, G., Grothendieck's theorem for operator spaces, Invent. Math., 150 (2002), 185-217.

[22] Smith, R. R., Completely bounded module maps and the Haagerup tensor product, $J$. Funct. Anal., 102 (1991), 156-175.

[23] Suen, C. Y., Completely bounded maps on $C^{*}$-algebras, Proc. Amer. Math. Soc., 93 (1985), 81-87.

[24] Takesaki, M., Theory of Operator Algebras I, Springer-Verlag, New-York, 1979.

[25] - Theory of Operator Algebras III, Springer-Verlag, New-York, 2002.

[26] Wittstock, G., Ein operatorwertiger Hahn-Banach Satz. (German) [An operator-valued Hahn-Banach theorem], J. Funct. Anal., 40 (1981), 127-150.

[27] Wysoczanski, J., Characterization of amenable groups and the Littlewood functions on free groups, Colloq. Math., 55 (1988), 261-265. 